\title{
Nonlinear Response of Silicon Double-Notch-Shaped Microdisk Resonators with Non-Evanescent Coupling
}

\author{
Hui Chen ${ }^{1}$, Chao Li ${ }^{2}$, Andrew W. Poon ${ }^{1}$, and H. K. Tsang ${ }^{2}$ \\ ${ }^{1}$ Photonic Device Laboratory, Department of Electronic and Computer Engineering \\ The Hong Kong University of Science and Technology \\ Clear Water Bay, Hong Kong SAR, China \\ Tel: (852)-2358-7905; Fax: (852)-2358-1485; Email: eeawpoon@ust.hk \\ ${ }^{2}$ Department of Electronic Engineering, The Chinese University of Hong Kong \\ Shatin, Hong Kong SAR, China
}

\begin{abstract}
We report double-notch-shaped microdisk resonators for silicon nonlinear-optic applications with direct in/out coupling via the microdisk notches. We measure optical bistability and resonance wavelength redshifts induced by the notch-coupled pump light.

(c) 2008 Optical Society of America

OCIS codes: (230.5750) Resonators, (130.4310) Integrated optics, Nonlinear
\end{abstract}

Recently, spiral-shaped microdisk resonators featuring a single notch for non-evanescent waveguide coupling has been demonstrated by one of us (AWP) on a silicon chip showing resonance Q of $\sim 10^{4}$ [1]. However, spiral-shaped microdisk resonators only allow non-evanescent coupling via a single notch. It is thus conceivable to employ two notches to form a double-notch-shaped microdisk resonator, which is potentially favorable for efficient optical in/out-coupling, particularly in the interest of nonlinear-optic applications. It has been known that silicon microdisk resonators exhibit nonlinear response in the input optical power range of $\mathrm{mW}$ [2], yet the evanescent side-coupling between the bus waveguide and the microdisk imposes precise waveguide fabrication or tapered-fiber positioning. We see that the double-notch-shaped microdisk offers an alternative microdisk structure with direct in/out-coupling via the notches and high-Q resonances. Here, we report our initial measurements of the nonlinear response of double-notch-shaped microdisk resonators.

Figures 1(a) and (b) show the scanning electron micrograph and the schematic of a waveguide-coupled doublenotch-shaped microdisk resonator. The microdisk comprises two hemi-circular disks with radii $R_{I}$ and $R_{2}$. The microdisk has a notch on each side with width of $w=R_{1}-R_{2}$. Our fabricated microdisk resonator on a silicon-oninsulator wafer (device height of $\sim 0.34 \mu \mathrm{m}$ ) shows resonance Q values on the order of $10^{4}$ using $R_{l}$ of $50 \mu \mathrm{m}$ and $w$ of $1 \mu \mathrm{m}$ (see Fig. 2(a)).

Figure 1(c) schematically shows the mechanism of a bistable transmission spectrum obtained by means of wavelength-increasing scanning. The circles represent the scanning wavelengths. When the wavelength is at offresonance, the optical power injected into the resonator is relatively low and thus the resonator has "cold" cavity resonance (solid-line). As the wavelength approaches the resonance lineshape, an increase in the cavity optical power redshifts the resonance wavelength (dashed-lines) due to thermo effects. Thus, the wavelength scan follows the blue side of the resonance lineshape until the wavelength reaches the redshifted resonance dip. A further increment in wavelength reaches the red side of the resonance lineshape, causing a drop in the cavity optical power. Consequently, the cavity resonance blueshifts and results in an abrupt transition from a low- to a high-transmission, signifying optical bistability [3].

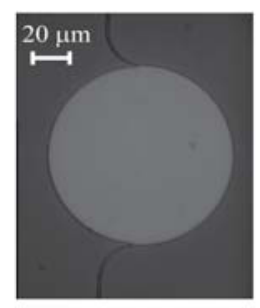

(a)

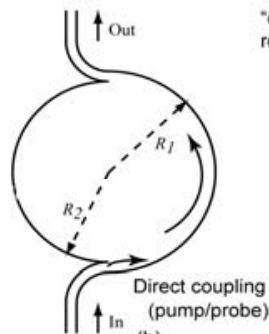

(b)

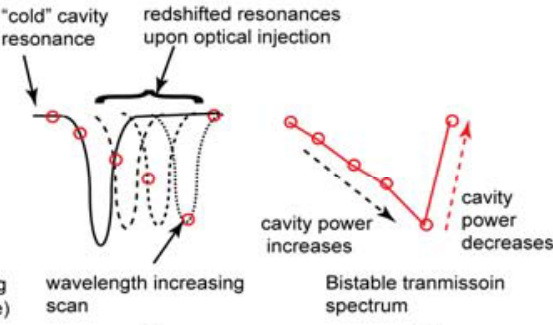

(d)

Fig 1. (a) Scanning electron micrograph of a waveguide-coupled double-notch-shaped microresonator $\left(R_{I} \sim 50 \mu \mathrm{m}, w \sim 0.4 \mu \mathrm{m}\right)$. (b) Schematic of a double-notch-shaped microresonator. (c) Mechanism of a bistable transmission spectrum obtained by means of wavelength-increasing scan. Circles: scanning wavelengths; Solid-line: "cold" cavity resonance; Dashed-lines: redshifted resonances upon injected optical power. (d) Schematic transmission spectrum obtained by means of wavelength-increasing scan. 


\section{CTuJ5.pdf}
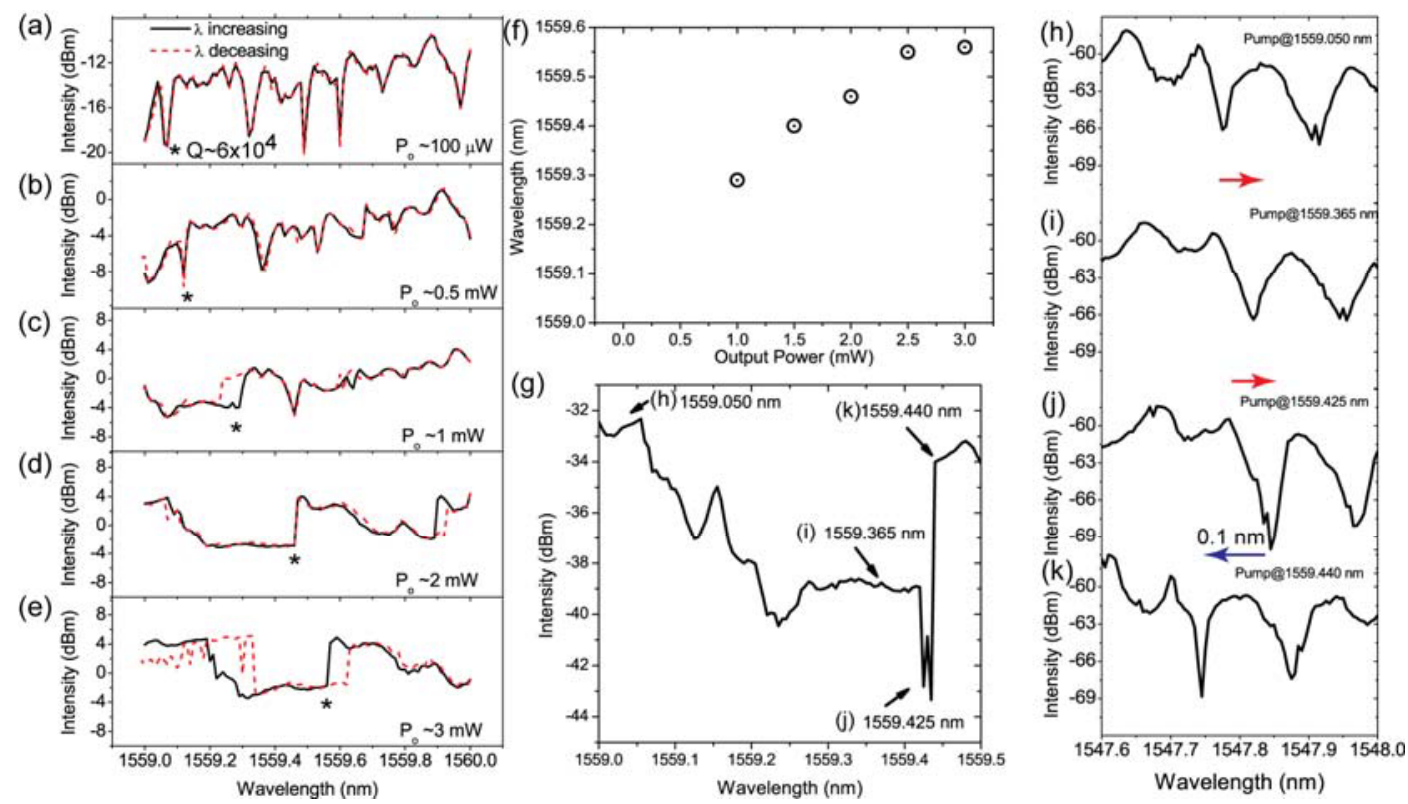

Fig 2. (a)-(e) Measured transmission spectra upon various input optical power of a double-notch-shaped microresonator $\left(R_{1} \sim 50 \mu \mathrm{m}, w \sim 1 \mu \mathrm{m}\right)$ for both wavelength increasing (solid-line) and wavelength decreasing scan (dashed-line). $P_{o}$ : output power. (f) Bistability transition wavelength for wavelength increasing scan as a function of output power. (g) Bistable transmission spectrum measured in wavelength increasing scan with the output power $\sim 2$ $\mathrm{mW}$. The labeled positions represent the pump wavelengths we perform the probe spectra measurements (h)-(k).

We measure the resonator transmission with the laser source scanning in both wavelength-increasing $(\uparrow \lambda)$ and wavelength-decreasing $(\downarrow \lambda)$ directions upon various optical input power (Fig. 2(a)-(e)). For transmission output power around $100 \mu \mathrm{W}$, both $\uparrow \lambda$ and $\downarrow \lambda$ scans show nearly identical transmission spectra of multimode resonances. The labeled resonance $(*)$ in Fig. 2(a) reveals a $\mathrm{Q}$ of $6 \times 10^{4}$. Further increment in the optical input power redshifts the resonance wavelength (Fig. 2(b)). As the output power reaches beyond $\sim 1 \mathrm{~mW}$, we observe significant resonance lineshape broadening (Figs. 2 (c)-(e)). The transmission spectra reveal a sharp transition at wavelength * from a low- to a high-transmission, which is consistent with Fig. 1(d). The $\downarrow \lambda$ scan reveals a transition wavelength distinct from the $\uparrow \lambda$ scan upon the same optical input power. Figure 2(f) shows the bistability transition wavelength in the $\uparrow \lambda$ scan redshifts with the waveguide optical output power.

In order to study the cavity response upon different pump wavelengths, we measure the transmission spectrum of a low-power probe wavelength that is arbitrarily offset from the pump wavelength $(\sim 1559 \mathrm{~nm})$. Figures 2(h)-(k) show the measured transmission spectra at the probe wavelength. Each spectrum is measured upon the pump wavelength approaching the labeled wavelength positions (shown in Fig. $2(\mathrm{~g})$ ) in $\uparrow \lambda$ scan. Figures 2(h)-(j) show the probe resonance redshifts as the pump wavelength scan from off-resonance (h) $1559.050 \mathrm{~nm}$ to on-resonance (j) $1559.425 \mathrm{~nm}$, indicating an increase in the injected optical power in the cavity. Figure 2(k) shows that the probe resonance blueshifts after the pump wavelength scan through the bistable transition wavelength at $\sim 1559.44 \mathrm{~nm}$, suggesting an abrupt decrease in the injected optical power. The probe resonances wavelength difference is around $0.1 \mathrm{~nm}$ when the pump scans from (j) $1559.425 \mathrm{~nm}$ to $(\mathrm{k}) 1559.440 \mathrm{~nm}$, indicating a refractive index change of $\sim 2 \times$ $10^{-4}$. The probe resonance lineshapes are also slightly broadened while the pump is at on-resonance wavelengths as a result of an increase in the nonlinear loss.

[1] J. Y. Lee, X. Luo and A. W. Poon, "Reciprocal transmission and asymmetric modal distributions in waveguide-coupled spiral-shaped microdisk resonators," Opt. Express 15, 14650-14666 (2007).

[2] M. Borsell, T. J. Johnson, and O. Painter, “Accurate measurement of scattering and absorption loss in microphotonic devices,” Opt. Lett. 32, 2954-2956 (2007).

[3] G. Priem, P. Dumon, W. Bogaerts, D. Van Thourhout, G. Morthier, and R. Baets, "Optical bistability and pulsating behaviour in silicon-oninsulator ring resonator structures," Opt. Express 13, 9623-9628 (2005). 